\title{
Comparative Analysis of Orthologous Genes between Anguina tritici, Ditylenchus destructor and Meloidogyne incognita
}

\author{
Ashish Kumar Singh ${ }^{1,2 *}$, Ila Joshi ${ }^{3}$, Kuldeep Kumar ${ }^{3}$, Amit Ahuja ${ }^{1}$ and Anil Sirohi ${ }^{1}$ \\ ${ }^{1}$ Division of Nematology, ICAR-Indian Agricultural Research Institute, \\ Pusa Campus, New Delhi-110012, India \\ ${ }^{2}$ Division of Crop protection, ICAR-Vivekananda Parvatiya Krishi AnusandhanSansthan, \\ Almora, Uttarakhand 263601, India \\ ${ }^{3}$ ICAR-National Institute for Plant Biotechnology, Pusa Campus, New Delhi, 110012, India \\ *Corresponding author
}

\section{A B S T R A C T}

\begin{tabular}{|c|}
\hline Keywords \\
\hline $\begin{array}{l}\text { Orthologous genes } \\
\text { Anguina tritici, } \\
\text { Ditylenchus } \\
\text { destructor and } \\
\text { Meloidogyne } \\
\text { incognita }\end{array}$ \\
\hline Article Info \\
\hline $\begin{array}{l}\text { Accepted: } \\
04 \text { September } 2020 \\
\text { Available Online: } \\
10 \text { October } 2020\end{array}$ \\
\hline
\end{tabular}

\section{Introduction}

Nematodes are the most abundant metazoans and highly diverse affecting human, animal and plant health and some are playing significant role in nutrient recycling and as successful bioagent of insect pest (Blaxter, 2003; Lambshead, 1993; Andrássy and Zombori, 1976). Among all the nematodes plant parasitic nematodes (PPNs) occupy significant posing due to their role in damaging crop and inflicting yield loss to wide range of crops (Singh et al., 2015).
Nematodes are very distinct organism due to its adaptability in diverse climatic condition and has been attributed due to morphological, behavioural and genetic plasticity (Coghlan, 2005).

The model nematode (Caenorhabditis elegans) was first nematode which genome sequenced in 1998 and given impetus for future endeavor to generate genomic information for other nematodes particularly for PPNs. Since then more than 100s of nematode genome have been sequenced and 
interpreted for diverse biological complexity and mystery of evolution. Related to evolutionary history of nematode that our understanding about the evolution of nematodes is enriching due to availability of genome sequences of wide range of nematodes including free living to parasite of plants (https://parasite.wormbase.org/index. html).

The available genomic datasets are ideal to investigate in depth about diverse biological phenomenon through comparative genomics tool. Comparative genomics among different feeding group of nematodes are important to unravel the force enabling them to feed on different parts of the plant despite their similar morphological architect. The mystery behind host suitability to adaptability to feed on different plant part, survivability lies in the genetic trait of nematode species. Although the genomic data are available on large scale on different database but analysing, investigating and drawing meaningful conclusion is still a challenge.

Comparative genomics is one of the approaches to predict the genes shared with different species and also to unravel the genes unique and novel to a particular species. The homologous genes (orthologue and paralogue) related to speciation or duplication are indispensable in tracing the evolutionary pattern of an organism and furthermore inferring orthology is most accurate alternative to describe similarities and differences in a genome (Gabaldon, 2013). Orthologs gene generally shows their syntenic relationship with closely related species and can be traced to a Comman ancestor during speciation events.

In contrast to orthologs, Paralogs show limited synteny, divergence in speciation and originated from duplication events in a species (Altenhoff and Dessimoz, 2012). The presence of similar orthologous sequences in different species indicates their similar biological function and contrary if sequence show greater divergence then other species may perform distinct function in particular species (Fitch, 2000).

In comparative genomics of two species, identification of orthologous genes and their degree of similarity between two species are the major steps in understanding evolutionary pattern of genes and genomes (Koonin et al., 1996). In the present study sequenced genome of seed gall nematode (A. tritici) and in its comparative genomics study enabled us for identification of orthologues genes, their function and evolution among different species of nematodes. In the present study we have used a graph-based method by utilizing Orthovenn 2 tool (Xu et al., 2019) for orthologous analysis to compare and annotate the orthologous cluster between the genome of Anguina tritici, Ditylenchus destructor and Meloidogyne incognita.

\section{Materials and Methods}

\section{Genome sequencing and assembly}

Recently we sequenced and assembled the draft genome of Anguina tritici (The gene bank submission will be available soon) using next generation sequencing approach. The obtained genomic data were screened for quality check and assembled using Abyss programme.

\section{Retrieving protein dataset of nematodes}

Protein sequence of $A$. tritici were predicted from de novo sequencing and assembly of genome using Trans Decoderprogramme (Haas et al., 2013). Protein sequence of $M$. incognita and $D$. destructor were downloaded from NCBI database (Zheng et al., 2016; Abad et al., 2008). 
Genome comparison between Anguina tritici, Ditylenchus destructor and Meloidogyne incognita

Genome of three nematode species namely $A$. tritici, $D$. destructor and $M$. incognita were compared using OrthoVenn 2.0 web server based on DIAMOND (v0.9.24) all against all comparison of nematode protein sequences. The orthologue clustering programme uses most popular heuristic best match approach for identification of orthologous genes conserved between two species. The programme was run after uploading each nematode protein sequences on web server with default parameter (with e-value 1e-5 and inflation value of 1.5) extended with pairwise heatmap. The obtained result can be accessed at supporting link 3

\section{Motif and phylogenetic analysis of Pectatelyase gene}

Annotated orthologue clusters using amigo gene ontology (Carbon et al., 2009) revealed various function among which the cluster 263 showed Pectatelyase activity in all three nematodes. Cluster 263 were selected for motif analysis using MEME version 4.12.0 (Bailey et al., 2009) and the protein hits were aligned using MUSCLE (Edgar et al., 2004).

\section{Results and Discussion}

In the recent past, genome sequencing efforts for nematodes have generated the huge amount of genomic dataset. Their comparative genomic analysis has revealed the origin of large number of genes through horizontal gene transfer events from bacteria have enabled the tylenchid nematode to parasitize plants (Mitreva et al., 2005). Therefore, genomic resources have been proven to be a potential resource for understanding of molecular mechanism, relations and evolutionary dynamics with other nematodes as well organism. In our effort to comparative genomics of $A$. tritici with other two nematodes hypothecated to find conserved and diverse genes among $A$. tritici, D. destructor and M. incognita.

Orthologous cluster analysis of three nematode species (A. tritici, D. destructor and $M$. incognita) using OrthoVenn 2.0 web server displayed comparison results in cluster table (Fig. 1 A, supporting link 1). The predicted summarized clustering result indicates column as species while each row of cell graphs an orthologous luster group. The programme predicted the presence of 12 number of cluster group (4 in each nematode species) and absence of cluster group in 9 cells (Fig. 1 A). The cumulative number of protein sequences predicted to be involved in cluster group of each nematode species were varied from 420-3453 (bar plot stacked at right in Fig. $1 \mathrm{~A}$ ). The $A$. tritici and $M$. incognita revealed to have low number of protein sequences (420) involved in cluster group in comparison to the A. tritici and $D$. destructor. Orthovenn displayed Venn diagram indicating the number of orthologues shared between the three species in which 3453 clusters were found to be shared between $A$. tritici, $D$. destructor and $M$. incognita.The obtained summary result suggested that a total of 22989 proteins, 6171 clusters and 10625 singletons were found in A. tritici which were to be highest in compassion to the other nematode species (Fig. 1 B).

The number of orthologue cluster shared between $A$. tritici and $D$. destructor were found to be highest (1726) than $D$. destructor vs $M$. incognita (764) and $A$. tritici vs $M$. incognita (420), Additionally, the Bar plot revealed the presence of total number of clusters were 6171, 6523 and 6301 respectively for $A$. tritici, D. destructor and M. incognita (Fig. 1 C). Pair wise heat map 
analysis revealed the overlapping cluster information in pairwise fashion among all the three-nematode species (Fig. 1 D, Table 1). Orthologues gene cluster shared to all the three nematodes were annotated to know their role in various biological process, molecular function and cellular component (Table 2, 3, 4 and Fig. 2). The details of annotations can be accessed at supporting link 2.

The annotation of clusters shared among all three nematode species revealed to have a suite of large number of genes playing variety of function in biological process such as host finding, parasitism, reproduction and survival. These genes were found to be identical to the $D$. destructor and M. incognita in comparison to the A. tritici (Abad et al., 2008, Szitenberg et al., 2017, Zheng et al., 2016). GO analysis reveals the most abundant number of GO were enriched for biological process, metabolic and cellular process (Table 2) and in case of molecular function GO terms significantly abundant for ion binding (Table 2).

Overall GO analysis revealed the largest number of GO terms was associated for biological process, cellular components and molecular functions. Other than shared comman clusters there were 572 clusters in $A$. tritici, 580 in D. destructor and 1664 in $M$. incognita as unique and specific to their origin (Fig. 1).

Table.1 Indicating the cluster number overlapped in genome of each nematode species in pair wise heat map

\begin{tabular}{|l|c|c|c|}
\hline Meloidogyne incognita & $\mathbf{3 8 7 3}$ & $\mathbf{4 2 1 7}$ & ----- \\
\hline Ditylenchus destructor & 5179 & ------ & 4217 \\
\hline Anguina tritici & & 5179 & 3873 \\
\hline & Anguina tritici & $\begin{array}{c}\text { Ditylenchus } \\
\text { destructor }\end{array}$ & $\begin{array}{c}\text { Meloidogyne } \\
\text { incognita }\end{array}$ \\
\hline
\end{tabular}

Table.2 Top 10 highest number of accessions for biological process

\begin{tabular}{|l|c|c|}
\hline Slimmed GO & Count of unique input accessions & Name \\
\hline GO:0008150 & 1602 & biological process \\
\hline GO:0008152 & 946 & metabolic process \\
\hline GO:0009987 & 939 & cellular process \\
\hline GO:0044237 & 690 & cellular metabolic process \\
\hline GO:0043170 & 678 & macromolecule metabolic process \\
\hline GO:0065007 & 657 & biological regulation \\
\hline GO:0044238 & 528 & primary metabolic process \\
\hline GO:0006807 & 441 & nitrogen compound metabolic process \\
\hline GO:0050896 & 413 & response to stimulus \\
\hline GO:0016070 & 399 & RNA metabolic process \\
\hline
\end{tabular}


Table.3 Top 10 highest number of accessions for Molecular function

\begin{tabular}{|l|c|c|}
\hline Slimmed GO & Count of unique input accessions & Name \\
\hline GO:0043167 & 48 & ion binding \\
\hline GO:0003674 & 41 & molecular function \\
\hline GO:0003676 & 27 & nucleic acid binding \\
\hline GO:0016787 & 25 & hydrolase activity \\
\hline GO:0005215 & 23 & transporter activity \\
\hline GO:0016740 & 20 & transferase activity \\
\hline GO:0005488 & 19 & binding \\
\hline GO:0016491 & 16 & oxidoreductase activity \\
\hline GO:0005515 & 12 & protein binding \\
\hline GO:0030234 & 10 & enzyme regulator activity \\
\hline
\end{tabular}

Table.4 Top 10 highest number of accessions for Cellular components

\begin{tabular}{|l|c|l|}
\hline Slimmed GO & Count of unique input accessions & Name \\
\hline GO:0005575 & 56 & cellular component \\
\hline GO:0016020 & 53 & membrane \\
\hline GO:0044464 & 26 & cell part \\
\hline GO:0005622 & 18 & intracellular \\
\hline GO:0043226 & 13 & organelle \\
\hline GO:0005634 & 10 & nucleus \\
\hline GO:0043229 & 10 & intracellular organelle \\
\hline GO:0005576 & 7 & extracellular region \\
\hline GO:0005739 & 6 & mitochondrion \\
\hline GO:0005856 & 4 & cytoskeleton \\
\hline
\end{tabular}

Fig.1 (A) Occurrence table indicating shared orthologue groups patterns between A. tritici, D. destructor and $M$. incognita. Left pattern indicating the species of nematode involved in clusters, shared cluster count among them and protein count among the shared clusters (B) cluster count in genome of each nematode species. Singleton describing those genes having no orthologue; (C) Venn diagram showing the shared orthologue clusters distribution among A. tritici, D. destructor and $M$. incognita (D) Pairwise heat map indicating the cluster number overlapped in genome of each nematode species

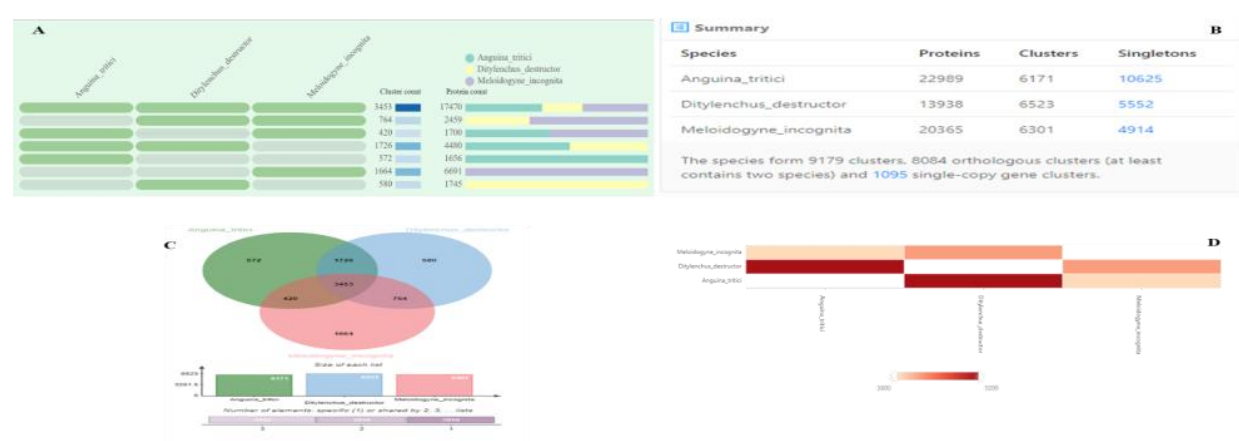


Fig.2 Details of Biological process, Molecular function and Cellular component

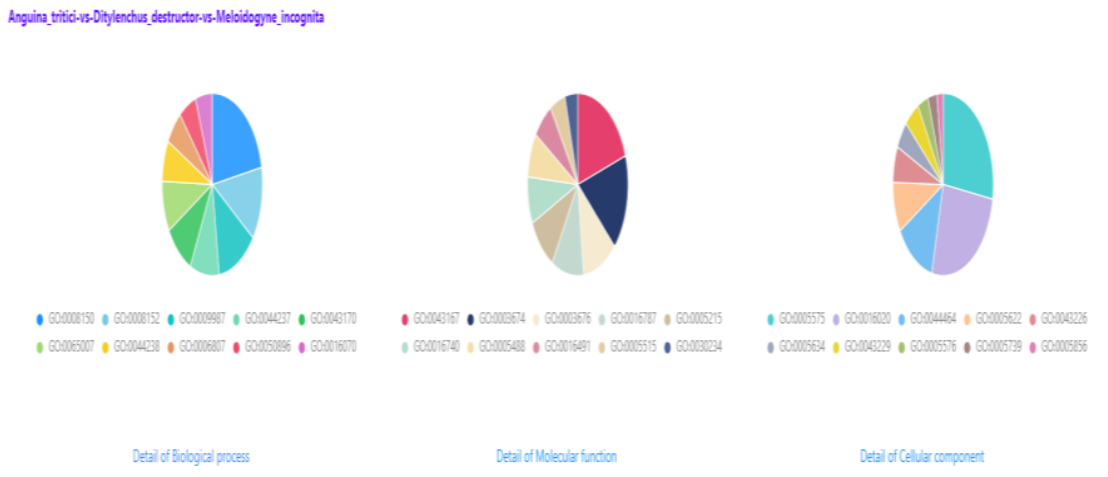

Fig.3 Annotation of cluster 63 showing Pectatelyase using different methods (A) protein network found with in cluster 63 among all three nematodes (B) Multiple sequence alignment of Pectatelyase protein sequence (C) Discovered Motifs in the protein sequence of cluster 263 with Pectatelyase function (D) Phylogenetic tree of Pectatelyase protein

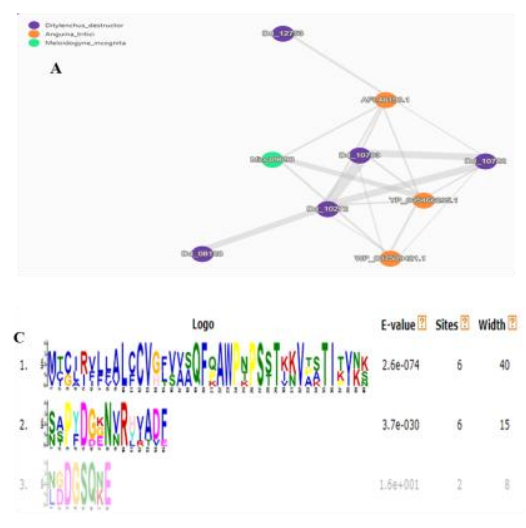

Unique gene identified in $A$. tritici was largely abundant for scavenger receptor activity, nuclease activity and post-embryonic development (supp. link 4), while for $D$. destructor it was for pathogenesis and mRNA processing (supp link 5). The most unique clustered were found for $M$. incognita were largely enriched for ubiquitin-dependent protein catabolic process and zinc ion binding (supp link 6). Taking all these into account it implies that in all these three nematode species $A$. tritici, D. destructor and $M$. incognita genome have their own uniqueness in terms of genetic component. Therefore, we hypothecate that the multiple reference genome for genomic analysis will be valuable for comparative genomic study of nematodes.

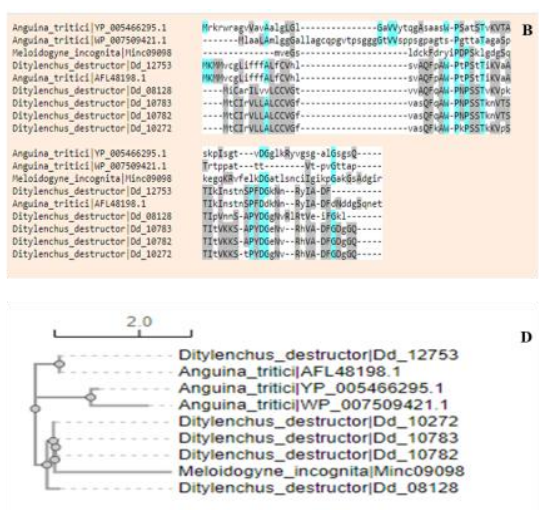

Among all these genes we selected significant GO term involved in breakdown of pectin (an essential component of defence layer in plant root) with annotated accession cluster 63, ID GO:0045490. This gene was found to be shared among all these three-nematode species. In host parasite interaction effectors play an important role in breakdown cell wall which is consist of pectin and cellulose (Davis et al., 2011). The presence of Pectatelyase in the $A$. tritici, D. destructor and $M$. incognita suggest that they might be playing similar functions. Conserved domain search for Pectatelyase protein comman in all three species revealed to have interlinking cluster and similarity with each other (Fig. 3 A, B). In this study identified $6,6,2$ sites for $A$. 
tritici, D. destructor and $M$. incognita respectively with e-cutoff value 1.6 to 3.6 (Fig. 3C). In order to investigate the evolutionary relationship and homology of Pectatelyase gene found in A. tritici, $D$. destructor and $M$. incognita we performed multiple sequence alignment (Fig. 3 B) and reconstructed phylogenetic tree (Fig. 3D). the obtained result indicated that the Pectatelyase gene has been expanded in A. tritici and $D$. destructor while in $M$. incognita it is in low number as compared to other two nematode species (Fig. 3D). Additionally, the Pectatelyase of A. triticifound to be closely related to $D$. destructor than $M$. incognita (Figure 1A).

Occurrence table indicating shared orthologue groups patterns between A. tritici, $D$. destructor and $M$. incognita. Left pattern indicating the species of nematode involved in clusters, shared cluster count among them and protein count among the shared clusters (B) cluster count in genome of each nematode species. Singleton describing those genes having no orthologue; (C) Venn diagram showing the shared orthologue clusters distribution among $A$. tritici, D. destructor and $M$. incognita (D) Pairwise heat map indicating the cluster number overlapped in genome of each nematode species

In conclusion the genome sequencing effort for nematodes have generated the huge amount of genomic dataset.Comparative genomics between $A$. tritici, D. destructor and $M$. incognita revealed various numerical variation in genetic contents, conservation of developmental related genes and many unique genes specific to all three nematode species exclusively.

\section{Supporting link}

1. Result page:https://orthovenn2.bioinfotoolkits.net /task/result/c5f9a05ef0e1341cab68133f77 $5 \mathrm{~d} 6 \mathrm{c} 2 \mathrm{~d}$

2. 2.https://orthovenn2.bioinfotoolkits.net/ov erlap/c5f9a05ef0e1341cab68133f775d6c2 $\mathrm{d} /$ jvennOverlap/ortho?bin $=\mathrm{QW}$ 5ndWluYV 90cml0aWNpLXZzLURpdHlsZW5jaHVz X2Rlc3RydWN0b3ItdnMtTWVsb2lkb2d5 bmVfaW5jb2duaXRh\&sp=QW5ndWluY V90cml0aWNpLERpdHlsZW5jaHVzX2R lc3RydWN0b3IsTWV sb2lkb2d5bmVfaW $5 \mathrm{jb} 2 \mathrm{duaXRh}$

3. https://orthovenn2.bioinfotoolkits.net/task/ result/c5f9a05ef0e1341cab68133f775d6c2 d

4. https://orthovenn2.bioinfotoolkits.net/over lap/c5f9a05ef0e1341cab68133f775d6c $2 d / j$ vennOverlap/ortho?bin=QW5ndWluYV90 cml0aWNp\&sp=QW5ndWluYV90cml0a WNpLERpdHlsZW5jaHVzX2Rlc3RydW N0b3IsTWVsb2lkb2d5bmVfaW5jb2duaX $\mathrm{Rh}$

\section{References}

Abad, P., Gouzy, J., Aury, J., Castagnone-Sereno, P., and Danchin, E. (2008). Genome sequence of the metazoan plant-parasitic nematode Meloidogyne incognita. Nat Biotechnol, 26.

Altenhoff and Dessimoz, 2012. A.M. Altenhoff, C. Dessimoz. Inferring orthology and paralogy. M. Anisimova (Ed.), Evolutionary Genomics: Statistical and Computational Methods, Springer Science.

Andrássy, I., and Zombori, L. (1976). Evolution as a basis for the systematization of nematodes. (London: Pitman).

Bailey, T. L., Boden, M., Buske, F. A., Frith, M., Grant, C. E., Clementi, L., Ren, J., Li, W. W., and Noble, W. S. (2009). MEME SUITE: tools for motif discovery and searching. Nucleic acids research, 37(Web Server issue), W202-W208.

Blaxter, M.L. (2003). Nematoda: genes, genomes and the evolution of parasitism. Adv. Parasitol. 54, 101-195.

Carbon, S., Ireland, A., Mungall, C. J., Shu, S., Marshall, B., Lewis, S., AmiGO Hub, and Web Presence Working Group (2009). 
AmiGO: online access to ontology and annotation data. Bioinformatics (Oxford, England), 25(2), 288-289.

Coghlan, A. Nematode genome evolution (2005), Worm Book, ed. The C. elegans Research Community, Worm Book,

Davis, E., Haegeman, A., Kikuchi, T. (2011) Degradation of the Plant Cell Wall by Nematodes. In: Jones J, Gheysen G, Fenoll $\mathrm{C}$, editors. Genomics and Molecular Genetics of Plant-Nematode Interactions: Springer Netherlands. pp. 255-272.

Edgar, R.C. (2004). MUSCLE: a multiple sequence alignment method with reduced time and space complexity. $B M C$ Bioinformatics 5, 113.

Fitch, W.M.(2000)Homology a personal view on some of the problems. Trends Genet; 16(5):227-31.

Gabaldón, T., Koonin, E.V. (2013) Functional and evolutionary implications of gene orthology. Nat Rev Genet. 2013; 14(5): 360-366.

Haas et al., (2013). de novo transcript sequence reconstruction from RNA-seq using the Trinity platform for reference generation and analysis. Nat Protoc. 2013 Aug; 8(8): 1494-512.

Howe, K. L., Bolt, B. J., Shafie, M., Kersey, P., and Berriman, M. (2017). Worm Base ParaSite - a comprehensive resource for helminth genomics. Molecular and biochemical parasitology, 215, 2-10.

Koonin, E.V., Mushegian, A.R., Bork, P. (1996)Non-orthologous gene displacement.
Trends Genet. 1996 Sep; 12(9):334-6

Lambshead, P.J. (1993). Recent developments in marine benthic biodiversity research. Oceanis 19, 5-24.

Mitreva, M., Blaxter, M.L., Bird, D.M., McCarter, J. P. (2005). Comparative genomics of nematodes, Trends in Genetics, Volume 21, Issue 10, Pages 573-581.

Singh, S. Singh, B. and Singh, A.P. 2015. Nematodes: A Threat to Sustainability of Agriculture, Procedia Environmental Sciences, Volume 29, Pp. 215-216.

Szitenberg A, Salazar-Jaramillo L, Blok VC, et al., Comparative Genomics of Apomictic Root-Knot Nematodes: Hybridization, Ploidy, and Dynamic Genome Change. Genome Biol Evol. 2017;9(10):2844-2861.

$\mathrm{Xu}$ et al., (2019). OrthoVenn2: a web server for whole-genome comparison and annotation of orthologous clusters across multiple species, Nucleic Acids Research, Volume 47, Issue W1, Pages W52-W58,

Zheng J, Peng D, Chen L, et al., The Ditylenchus destructor genome provides new insights into the evolution of plant parasitic nematodes. Proc Biol Sci. 2016; 283(1835): 20160942.

Zheng, J., Peng, D., Chen, L., Liu, H., Chen, F., $\mathrm{Xu}$, M., Sun, M. (2016). The Ditylenchus destructor genome provides new insights into the evolution of plant parasitic nematodes. Proceedings of the Royal Society B: Biological Sciences, 283(1835), 20160942

\section{How to cite this article:}

Ashish Kumar Singh, Ila Joshi, Kuldeep Kumar, Amit Ahuja and Anil Sirohi. 2020. Comparative Analysis of Orthologous Genes between Anguina tritici, Ditylenchus destructor and Meloidogyne incognita. Int.J.Curr.Microbiol.App.Sci. 9(10): 391-398. doi: https://doi.org/10.20546/ijcmas.2020.910.048 